\title{
Powerful Raman Lidar systems for atmospheric analysis and high-energy physics experiments
}

\author{
George Avdikos ${ }^{\mathrm{a}}$ \\ Raymetrics S.A., 12 Spartis, 14452 Metamorfosis, Greece
}

\begin{abstract}
In this paper the author presents modern commercial Raman Lidar systems which can be applied to high-energy physics experiments. Raymetrics is a world-leader in laser remote (lidar) sensing applications. Products series include lidar systems for various applications like atmospheric analysis, meteorology, and recently more operational applications including volcanic ash detection systems, visual rangers for application to airports etc.
\end{abstract}

\section{Introduction}

The Raymetrics Raman Lidar systems are active laser remote sensing instruments that measure the vertical profile of suspended aerosols and atmospheric molecules. High spatial and temporal resolution means that our Lidar systems are powerful tools for monitoring the evolution of a range of meteorological and atmospheric parameters. Raymetrics Lidar systems are capable of detecting the Raman shifted backscattered radiation from atmospheric Nitrogen at $387 \mathrm{~nm}, 607 \mathrm{~nm}$ and water vapour at $408 \mathrm{~nm}$, and the Mie/Rayleigh backscattering radiation from atmospheric gases, aerosol particles and molecules. The use of a Raman Lidar is a well-established method of determining the vertical profile of the aerosol extinction coefficient independently from the aerosol backscatter coefficient, resulting in a dramatic reduction in the associated uncertainties.

\section{Raman Lidars}

Raymetrics lidars are suitable for a range of atmospheric research, including studies/applications of:

Aerosols - Vertical and temporal profiling, Atmospheric layering, Atmospheric transport studies, Location of pollution emission sources.

Clouds - Water/ice determination, Cloud dynamic evolution, Cloud thicknesses (thin clouds), 3D dynamic cloud modelling, Cloud base height, Convective cell structure.

Planetary Boundary Layer - PBL height, Layer mixing height, Dynamic structure evolution, Water Vapour - water vapour Raman LIDAR required.

Ozone - Custom DIAL Ozone LIDAR required.

The Raymetrics Raman Lidar system includes:

- Laser emitter based on the fundamental, second and third harmonic frequency of a compact, pulsed, solid state $\mathrm{Nd}: Y A G$ laser.

\footnotetext{
a e-mail: gavdikos@raymetrics.gr
}

- Optical telescope and receiving optics of an advanced design and superior performance.

- Sophisticated computer-aided opto-mechanical design applied to all components, keeping the overall size and weight of the LIDAR systems to a minimum and guaranteeing the reliable operation of the system.

- Data acquisition system especially designed for optical signal detection. Simultaneous analogue detection of the photo-multiplier current and single photon counting are integrated into the same acquisition system, substantially increasing the dynamic range of the acquired signal compared to conventional systems.

- Independent detection channels equipped with dedicated subminiature photomultiplier tubes, selected for their excellent time and spectral response to each individual wavelength.

- Complete hardware and software solution.

\subsection{Vertical Multi-wavelength Raman Lidars}

Raymetrics offers a range of highly customizable vertical LIDARs. At the highest end of the scale is the LR321D400 Raman Depolarization LIDAR, which comes with an extremely powerful laser emitting $400 \mathrm{~mJ}$ per pulse, plus a large telescope with a $400 \mathrm{~mm}$ diameter. The system is however fully customizable with different wavelengths $(355 \mathrm{~nm}$ co-polarization, $355 \mathrm{~nm}$ crosspolarization, $387 \mathrm{~nm}$ nitrogen Raman, $408 \mathrm{~nm}$ water vapour Raman, $532 \mathrm{~nm}$ co-polarization, $532 \mathrm{~nm}$ crosspolarization, $607 \mathrm{~nm}$ nitrogen Raman, $1064 \mathrm{~nm}$ backscatter), different telescope sizes $(200,300$ or $400 \mathrm{~mm}$ as standard, others on request), and different laser energies $(100,200$ or $300 \mathrm{~mJ})$. Other customization options such as hatches, blowers, GPS, etc, are available on request.

\subsection{Scanning Raman Lidar}

Raymetrics's 3D scanning LIDAR comes with a $200 \mathrm{~mm}$ telescope and a laser emitting at $30 \mathrm{~mJ}$ per pulse at $355 \mathrm{~nm}$ as standard. Operating in the UV range with $10 \mathrm{X}$ beam expansion manes that the system is eyesafe even with such high energy emission (eye-safety is defined as according to the EU standard on laser safety EN 60825-1:2007). The system can detect up

This is an Open Access article distributed under the terms of the Creative Commons Attribution License 4.0, which permits unrestricted use, distribution, and reproduction in any medium, provided the original work is properly cited. 


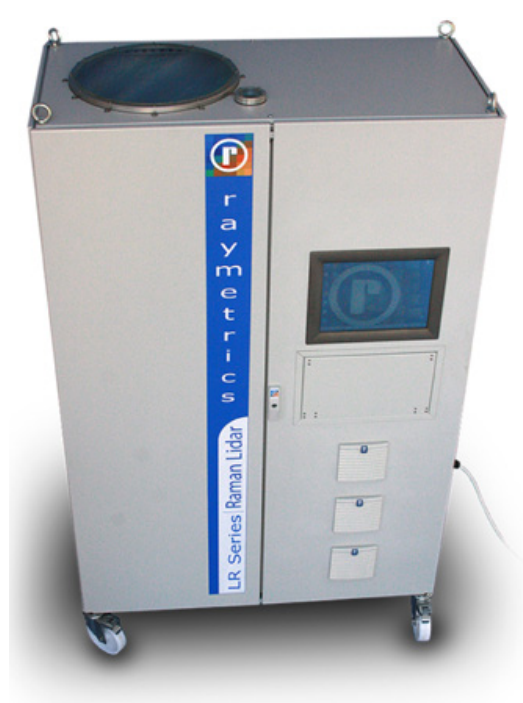

Figure 1. Raymetrics multi-wavelength Raman lidar system.

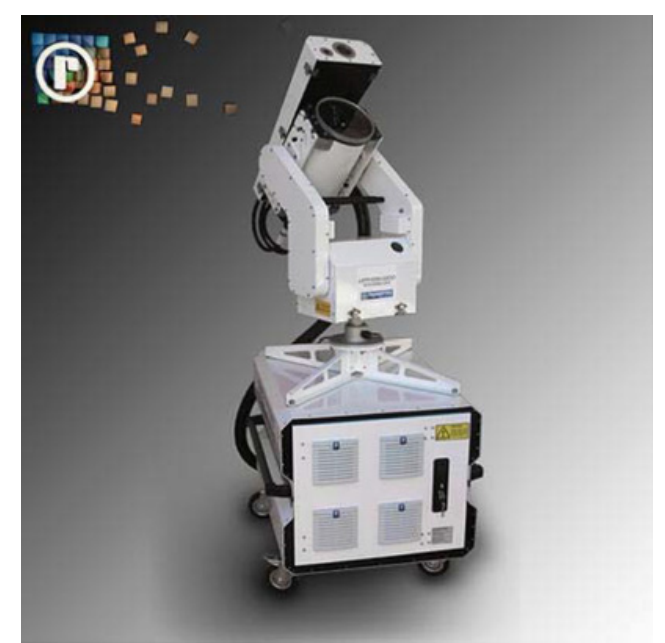

Figure 2. Raymetrics scanning Raman lidar system.

to 3 channels ( $355 \mathrm{~nm}$ co-polarization, $355 \mathrm{~nm}$ crosspolarization, $387 \mathrm{~nm}$ nitrogen Raman) and can be used for a variety of purposes, including pollution and dust tracking, aerosol characterization, and remote visibility measurement.

A choice of deployment types is also available:

- As standard, Raymetrics model LR111-ESS-D200 LIDARs now come in fully self-contained units (Fig. 2). System rotates with the help of a fully-automated scanning mechanics allowing the monitoring of the atmospheric aerosols in three dimensions (3D).

- For telescopes larger than $200 \mathrm{~mm}$, a manual rotation mechanism can also be used instead of the standard automated scanner (Fig. 3). This allows the user to manually turn a wheel to point the LIDAR in any direction required.

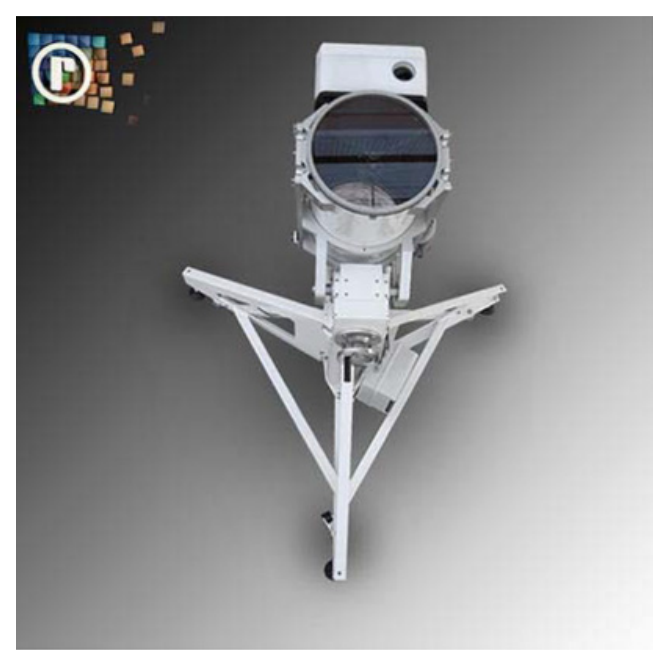

Figure 3. Raymetrics manually-rotated Raman lidar system.

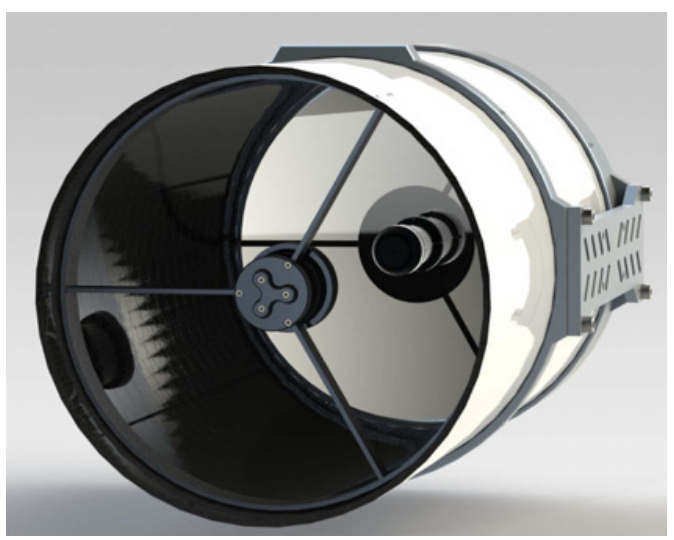

Figure 4. Receiving Telescope Design Example.

\section{Sophisticated Opto-mechanical design}

Transmission side: The Transmitter consists of two subunits

i) Laser System.

ii) Beam Expanders and Reflective Mirrors.

These units are integrated into the Lidar system and arrive fully pre-configured.

i) Laser: the laser is a true "turn-key" system shipped in fully operable form. No adjustments are necessary to make the system fully operational. The cable and coolant interconnects are labeled for simplicity and are of the quick-connect type. Hardware interlock and safety features are included in the electronics and cooling units to ensure hookups are secure and complete before the laser can be operated.

ii) Beam Expander Unit and Reflective Mirrors: the Beam Expander Unit (BEXP) is designed to provide a factory-set expansion of the laser beam at the wavelengths selected for the Lidar system. These units are integrated into the Lidar system and arrive fully pre-configured for optimum performance. The beam expander eliminates any 


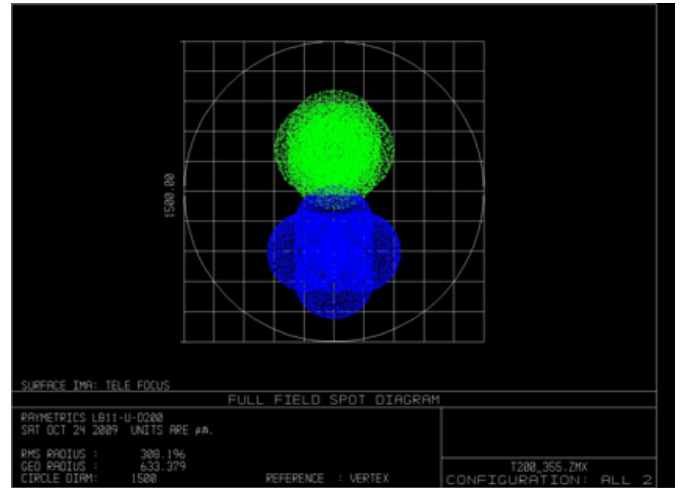

Figure 5. Near Field and Far Field Spot Diagrams at Field Stop (performed with ZEMAX [1]).

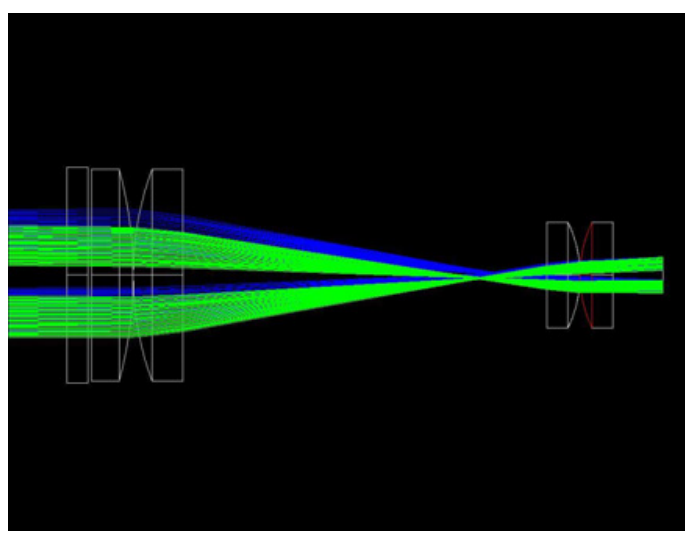

Figure 6. Eyepiece Optical Design and Performance.

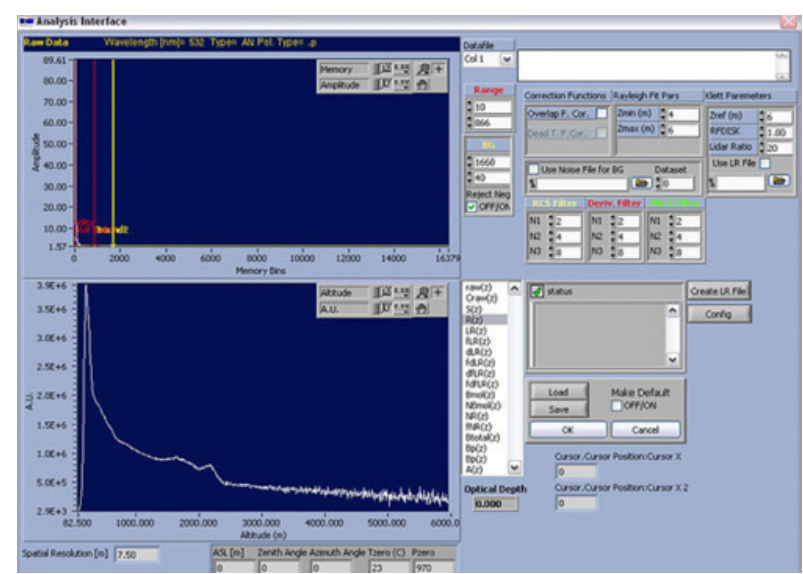

Figure 7. Data analysis software.

difference in the divergence of the different wavelengths, resulting in error-free measurements at all wavelengths from the near field up to the far field. The beam shifter is comprised of a pair of $\mathrm{HR}$ reflective mirrors mounted at a $45^{\circ}$ angle on a high precision $\mathrm{CNC}$ machined component which moves the beam $15 \mathrm{~mm}$. This ensures that no reflections will return into the laser and cause any permanent damage. In addition it rejects any possible residuals in the green or infrared ranges.

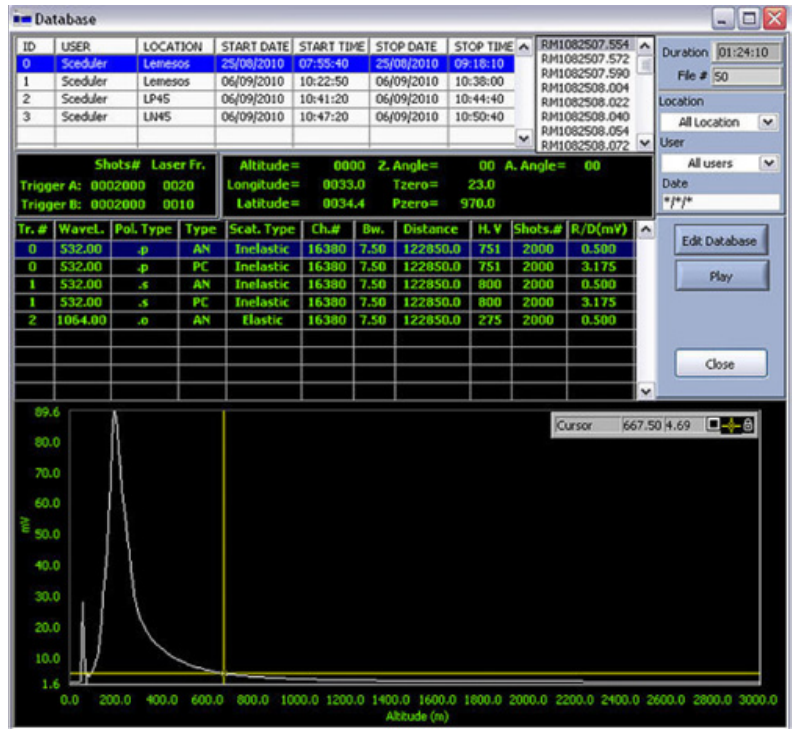

Figure 8. Database for data archiving.

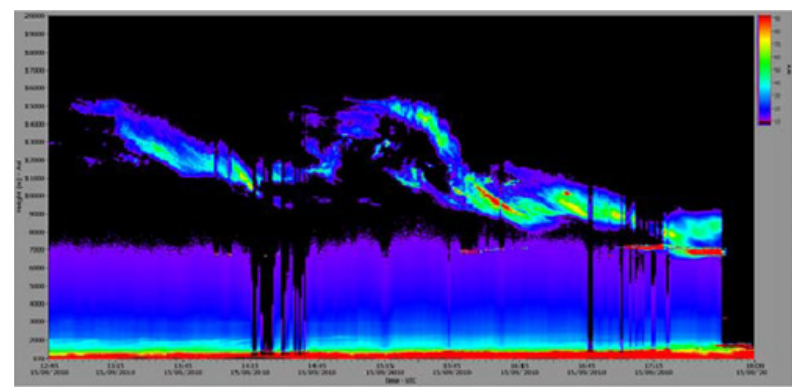

Figure 9. Vertical real-time data - clouds at $>15 \mathrm{~km}$.

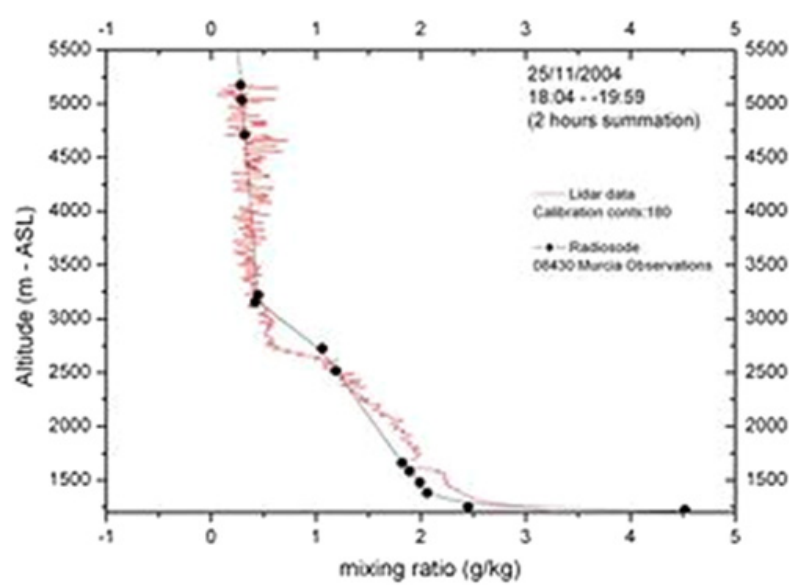

Figure 10. Comparison with radiosonde data.

Receiver side: The receiving system consists of four subunits:

- Receiving Telescope.

- Wavelength Separation Unit (WSU).

- Detectors.

- Data Acquisition Unit.

i) Receiving Telescope: The telescope and optical systems of all Raymetrics Lidars are fully designed in-house (Fig. 4). This ensures that the Lidar telescope is optimized 
for Lidar applications. Raymetrics does not use "off-theshelf" telescopes which have been designed for other applications. The receiving telescope of the Lidar system is a Cassegrainian design. The primary reflective mirror has a diameter of $\mathbf{4 0 0} \mathbf{~ m m}$. The secondary and primary mirrors are coated with a durable high reflective coating perfect for the 355-1064 $\mathbf{~ n m ~ s p e c t r a l ~ r e g i o n . ~ T h e ~ o p t i c a l ~}$ material selected shows a very low thermal expansion coefficient. All telescopes are fully designed through extensive raytracing techniques which guarantee the best performance at the selected wavelengths and operational ranges [1]. Received Lidar beams are collected and focused on an Optical Unit (OU) placed at the telescope's focal point. A calibrated ring iris diaphragm is used as a field stop, so the user can easily modify the field of view of the telescope for exceptional control of range and resolution ( $1 \mathrm{~mm}$ to $12 \mathrm{~mm}$ diameter) (Fig. 5).

ii) Wavelength Separation Unit (WSU) At the entrance of the Wavelength Separation Unit (WSU) of the Lidar system the received beams are collimated to one parallel beam. A series of factory preset custom-made dichroic reflective mirrors perform the wavelength separation at the various wavelengths. For each detected wavelength, an appropriate eye-piece is designed which ensures correct focus on the PMT active area (Fig. 6).

iii) Detectors - Signals inside the WSU are detected by avalanche photodiodes (APDs) directly mounted at the respective exits of the WSU. All PMTs/APDs used in Raymetrics Lidar systems are chosen for their excellent sensitivity, low offsets and small size

- Typical Detectors: HAMAMATSU R7880 series.

- All detectors come with a specially designed eye-piece which ensures the best performance from near field up to far field, eliminating any unwanted effect of possible inhomogeneity of the cathode.

iv) Data Acquisition Unit (DAU) The Transient Recorder integrated into Raymetrics Lidar systems within the Control Unit is a powerful data acquisition system manufactured by LICEL [2]. It has been designed specifically for remote sensing applications. The combination of a powerful $\mathrm{A} / \mathrm{D}$ converter with a fast photon counting system increases the dynamic range of the acquired signal substantially compared to conventional systems. A high-speed data interface to the host computer allows readout of the acquired signal even between two laser shots.

\section{Software GUI, processing - Data examples}

Raymetrics provides a full suite of custom-designed LIDAR software in a common GUI with every system, including modules for: System Alignment, Measurement Scheduling, Data Acquisition, Data Storage (Database), Data Analysis, Data Visualization, System Diagnostics (Figs. 7, 8).

Raymetrics software analysis module gives the possibility of combining together the signals detected in analog and photon-counting mode, resulting in an excellent signal both close by and far away from the Lidar system. Analysis of Lidar data is performed using three different algorithms: Klett algorithm [3] and Raman algorithm [4]. The temperature profile can be retrieved using the Raman algorithm described in [5] (Figs. 9, 10).

\section{References}

[1] www.zemax.com

[2] WwW.licel.com

[3] J.D. Klett, Applied Optics 24, 1638 (1985)

[4] A. Ansmann et al., Applied Optics 31, pp. 7113 (1992)

[5] Andreas Behrendt et al., Applied Optics, 41, pp. 7657 (2002) 